\title{
AMALGAMATED FREE PRODUCT OVER CARTAN SUBALGEBRA, II SUPPLEMENTARY RESULTS \& EXAMPLES
}

\author{
YOSHIMICHI UEDA
}

\section{INTRODUCTION}

Let $A \supseteq D \subseteq B$ be two von Neumann algebras together with a common Cartan subalgebra. Then the amalgamated free product $M=A *_{D} B$ with respect to the unique conditional expectations from $A, B$ onto $D$ can be considered. In our previous paper [U1], the questions of its factoriality and type classification were discussed in detail, which will be reviewed in $\S 4$. The main purpose of the paper is to give further supplementary results obtained after the completion of the previous paper together with discussing some examples.

The author would like to express his sincere gratitude to the organizers Bruce Blackadar \& Hideki Kosaki for inviting him to the US-Japan seminar 1999 held at Fukuoka, Japan and for giving this opportunity to present this paper.

\section{Amalgamated Free Products of von Neumann algebras}

Let $A \supseteq D \subseteq B$ be $\sigma$-finite von Neumann algebras, and let $E_{D}^{A}: A \rightarrow D$, $E_{D}^{B}: B \rightarrow D$ be faithful normal conditional expectations. Then one can consider the amalgamated free product of $A$ and $B$ over $D$ with respect to the conditional expectations $E_{D}^{A}, E_{D}^{B}$ :

$$
\left(M, E_{D}^{M}\right)=\left(A, E_{D}^{A}\right) *_{D}\left(B, E_{D}^{B}\right),
$$

which is defined as a pair of a von Neumann algebra $M$ into which the triple $A \supseteq$ $D \subseteq B$ is embedded and a faithful normal conditional expectation $E_{D}^{M}: M \rightarrow D$, and characterized by the following three conditions:

- $M$ is generated by the subalgebras $A, B$;

- $\left.E_{D}^{M}\right|_{A}=E_{D}^{A},\left.E_{D}^{M}\right|_{B}=E_{D}^{B}$

- $A, B$ are free with amalgamation over $D$ in the $D$-probability space $(M \supseteq$ $\left.D, E_{D}^{M}\right)$, see [VDN], i.e.,

$$
E_{D}^{M}\left(\left\{\text { alternating words in } A^{\circ}, B^{\circ}\right\}\right)=0,
$$

2000 Mathematics Subject Classification. Primary 46L54 Secondary 37A20. 
where we denote $A^{\circ}=\operatorname{Ker} E_{D}^{A}, B^{\circ}=\operatorname{Ker} E_{D}^{B}$ as usual.

For the details, we refer to [P2], [VDN],[U1] (see also [BD]).

In analysis on type III factors, modular automorphisms are of central importance so that we need to compute the modular automorphisms $\sigma_{t}^{\varphi \circ E_{D}^{M}}(t \in \mathbf{R})$ for a faithful normal state $\varphi$ on $D$.

Theorem 2.1. ([U1, Theorem 2.6]) We have

$$
\left.\sigma_{t}^{\varphi \circ E_{D}^{M}}\right|_{A}=\sigma_{t}^{\varphi \circ E_{D}^{A}},\left.\quad \sigma_{t}^{\varphi \circ E_{D}^{M}}\right|_{B}=\sigma_{t}^{\varphi \circ E_{D}^{B}} .
$$

The modular operator $\Delta_{\varphi \circ E_{D}^{M}}$ and the modular conjugation $J^{M}$ can be also computed explicitly. (See [U1, Appendix I].)

\section{Amalgamated Free Products over Cartan Subalgebras}

Let $A$ and $B$ be von Neumann algebras with separable preduals, and we suppose that they have a common Cartan subalgebra $D$ or equivalently that there is a common subalgebra $D$ satisfying:

- $D$ is a MASA in both $A$ and $B$;

- there are (automatically unique faithful) normal conditional expectations $E_{D}^{A}: A \rightarrow D, E_{D}^{B}: B \rightarrow D$

- the normalizers $\mathcal{N}_{A}(D), \mathcal{N}_{B}(D)$ generate the whole $A, B$, respectively.

(See $[\mathrm{FM}]$.) Let

$$
\left(M, E_{D}^{M}\right)=\left(A, E_{D}^{A}\right) *_{D}\left(B, E_{D}^{B}\right),
$$

and we will write $M=A *_{D} B$ since there is no other choice of normal conditional expectations from $A, B$ onto $D$.

The triple $A \supseteq D \subseteq B$ produces two countable non-singular Borel equivalence relations $\mathcal{R}_{A}, \mathcal{R}_{B}$ over a common standard Borel probability space $(X, \mu)$ in such a way that

$$
A=W_{\sigma_{A}}^{*}\left(\mathcal{R}_{A}\right), \quad B=W_{\sigma_{B}}^{*}\left(\mathcal{R}_{B}\right), \quad D=L^{\infty}(X, \mu),
$$

where $W_{\sigma_{A}}^{*}\left(\mathcal{R}_{A}\right), W_{\sigma_{B}}^{*}\left(\mathcal{R}_{B}\right)$ denote the von Neumann algebras constructed from $\mathcal{R}_{A}, \mathcal{R}_{B}$ together with relevant 2-cocycles $\sigma_{A}, \sigma_{B}$, respectively, by the FeldmanMoore construction $([\mathrm{FM}])$. After fixing a point realization $D=L^{\infty}(X, \mu)$, such a pair $\left(\mathcal{R}_{A}, \mathcal{R}_{B}\right)$ is uniquely determined up to null set. (This fact will be discussed in [U2] in detail.) Therefore, the countable non-singular Borel equivalence relation

$$
\mathcal{R}_{M}:=\mathcal{R}_{A} \vee \mathcal{R}_{B}(\subseteq X \times X)
$$

is a canonical object attached to the triple $A \supseteq D \subseteq B$, and we call this equivalence relation the canonical equivalence relation associated with the amalgamated free product $M=A *_{D} B$ or the triple $A \supseteq D \subseteq B$. 


\section{Factoriality \& Type Classification ([U1])}

In this section, we review our previous paper [U1].

Let $A \supseteq D \subseteq B$ be as in $\S 3$, i.e., two von Neumann algebras (with separable preduals) and a common Cartan subalgebra. We here discuss the amalgamated free product $M=A *_{D} B$. The first problem is its factoriality, namely, to find a suitable sufficient condition for the amalgamated free product $M$ to be a factor. A satisfactory answer to the problem was given in our previous paper.

Theorem 4.1. ([U1, Theorem 4.3]) If either $A$ or $B$ is a factor of non-type I, then the amalgamated free product $M=A *_{D} B$ over a common Cartan subalgebra $D$ becomes a factor. More precisely, if $A($ or $B)$ is a factor of non-type I, then there is a faithful normal state $\varphi$ on $D$ such that

$$
\left(A_{\varphi \circ E_{D}^{A}}\right)^{\prime} \cap M \subseteq A \quad\left(\operatorname{resp} .\left(B_{\varphi \circ E_{D}^{B}}\right)^{\prime} \cap M \subseteq B\right) .
$$

Furthermore, if $A$ (or $B)$ is further assumed to be of type $\operatorname{III}_{\lambda}(0<\lambda \leq 1)$, then the state $\varphi$ can be chosen in such a way that

$$
\left(A_{\varphi \circ E_{D}^{A}}\right)^{\prime} \cap A=\mathbf{C} 1 \quad\left(\operatorname{resp} .\left(B_{\varphi \circ E_{D}^{B}}\right)^{\prime} \cap B=\mathbf{C} 1\right) .
$$

This result can be generalized further. Such a generalization will be discussed later, see Remark 4.8 (2),(3).

The second problem seems Murray-von Neumann-Connes' type classification of the amalgamated free product $M$. In this direction, we obtained the following corollaries of Theorem 4.1:

Corollary 4.2. [U1, Corollary 4.5]) Suppose that both $A$ and $B$ are factors of non-type I. If $M=A *_{D} B$ is of type $\mathrm{III}_{0}$, then both $A$ and $B$ must also be of type $\mathrm{III}_{0}$.

Corollary 4.3. ([U1, p.377]) Suppose that both $A$ and $B$ are factors of non-type I.

(1) If either $A$ or $B$ of type $\mathrm{II}_{1}$ and if $M=A *_{D} B$ is semi-finite (i.e., has a faithful semi-finite normal trace), then $M$ must be of type $\mathrm{II}_{1}$.

(2) If either $A$ or $B$ is of type $\operatorname{III}_{\lambda}(0<\lambda<1)$, then $M=A *_{D} B$ must be of type $\mathrm{III}_{\lambda^{1 / n}}$ or of type $\mathrm{III}_{1}$.

(3) If either $A$ or $B$ is of type $\mathrm{III}_{1}$, then $M=A *_{D} B$ must be of type $\mathrm{III}_{1}$.

(4) If $A$ is of type $\mathrm{III}_{\lambda}$ and $B$ of type $\mathrm{III}_{\mu}$ with $\frac{\log \lambda}{\log \mu} \notin \mathbb{Q}$, then $M=A *_{D} B$ must be of type $\mathrm{III}_{1}$. 
For a while, we assume that $A \supseteq D \subseteq B$ is a general triple of $\sigma$-finite von Neumann algebras together with faithful normal conditional expectations $E_{D}^{A}$ : $A \rightarrow D, E_{D}^{B}: B \rightarrow D$, and let

$$
\left(M, E_{D}^{M}\right)=\left(A, E_{D}^{A}\right) *_{D}\left(B, E_{D}^{B}\right)
$$

be the amalgamated free product. Choose and fix a faithful normal state $\varphi$ on $D$, and we set:

$$
\widetilde{A}:=A \rtimes_{\sigma^{\varphi \odot E_{D}^{A}}} \mathbf{R} \supseteq \widetilde{D}:=D \rtimes_{\sigma^{\varphi}} \mathbf{R} \subseteq \widetilde{B}:=B \rtimes_{\sigma^{\varphi \odot E_{D}^{B}}} \mathbf{R},
$$

and

$$
\widetilde{M}:=M \rtimes_{\sigma^{\varphi \circ E_{D}^{M}}} \mathbf{R} .
$$

Then there are faithful normal conditional expectations

$$
\begin{array}{ll}
\widehat{E_{D}^{A}}: \widetilde{A} \rightarrow \widetilde{D} ; \quad \widehat{E_{D}^{A}}\left(\int_{-\infty}^{\infty} a(t) \lambda(t) d t\right):=\int_{-\infty}^{\infty} E_{D}^{A}(a(t)) \lambda(t) d t, \\
\widehat{E_{D}^{B}}: \widetilde{B} \rightarrow \widetilde{D} ; & \widehat{E_{D}^{B}}\left(\int_{-\infty}^{\infty} b(t) \lambda(t) d t\right):=\int_{-\infty}^{\infty} E_{D}^{B}(b(t)) \lambda(t) d t, \\
\widehat{E_{D}^{M}}: \widetilde{M} \rightarrow \widetilde{D} ; & \widehat{E_{D}^{M}}\left(\int_{-\infty}^{\infty} m(t) \lambda(t) d t\right):=\int_{-\infty}^{\infty} E_{D}^{M}(m(t)) \lambda(t) d t .
\end{array}
$$

Theorem 4.4. ([U1, Theorem 5.1]) In the current general setting, we have

$$
\left(\widetilde{M}, \widehat{E_{D}^{M}}\right) \cong\left(\widetilde{A}, \widehat{E_{D}^{A}}\right) *_{\widetilde{D}}\left(\widetilde{B}, \widehat{E_{D}^{B}}\right)
$$

Moreover, the dual action $\theta_{t}^{M}(t \in \mathbf{R})$ associated with $M$ is determined by those $\theta_{t}^{A}, \theta_{t}^{B}$ associated with $A, B$, respectively, in such a way that

$$
\left.\theta_{t}^{M}\right|_{\widetilde{A}}=\theta_{t}^{A},\left.\quad \theta_{t}^{M}\right|_{\widetilde{B}}=\theta_{t}^{B} .
$$

Let us return to the original setting, namely, the triple $A \supseteq D \subseteq B$ consists of two von Neumann algebras with separable preduals and a common Cartan subalgebra. By Theorem 4.4, we have

$$
\widetilde{M}\left(=\widetilde{A *_{D} B}\right) \cong \widetilde{A} *_{\widetilde{D}} \widetilde{B} .
$$

Since $\widetilde{D}$ is also a common Cartan subalgebra in both $\widetilde{A}$ and $\widetilde{B}$, we write $\widetilde{A} * \widetilde{D} \widetilde{B}$ as the amalgamated free product von Neumann algebra of $\widetilde{A}$ and $\widetilde{B}$ over $\widetilde{D}$ with respect to the conditional expectations $\widehat{E_{D}^{A}}, \widehat{E_{D}^{B}}$ since no confusion is possible.

We further suppose that both $A$ and $B$ are factors of non-type I in what follows. Theorem 4.4 (or (4.5)) together with the proof of Theorem 4.2 implies 
Theorem 4.5. ([U1, Theorem 5.4]) In the current setting, we have

$$
\mathcal{Z}(\widetilde{M})=\mathcal{Z}(\widetilde{A}) \cap \mathcal{Z}(\widetilde{B}) \subseteq \widetilde{D}
$$

Let $\left(X_{A}, F_{t}^{A}\right),\left(X_{B}, F_{t}^{B}\right)$ be the flows of weights $([\mathrm{CT}])$ of $A, B$, respectively. Fix a point realization $D=L^{\infty}(X, \mu)$, and set $X_{D}:=X \times \mathbf{R}$ equipped with the usual product measure $d \mu \otimes e^{-t} d t$ and $F_{t}^{D}(x, s):=(x, s+t)$. Then there are two factor maps

$$
\pi_{A}^{D}:\left(X_{D}, F_{t}^{D}\right) \rightarrow\left(X_{A}, F_{t}^{A}\right), \quad \pi_{B}^{D}:\left(X_{D}, F_{t}^{D}\right) \rightarrow\left(X_{B}, F_{t}^{B}\right)
$$

since $\widetilde{D}$ is a common Cartan subalgebra in both $\widetilde{A}, \widetilde{B}$. Let $\left(X_{M}, F_{t}^{M}\right)$ be the flow of weights of $M$. Theorem 4.5 says that there are three factor maps

$$
\begin{aligned}
\pi_{M}^{A}:\left(X_{A}, F_{t}^{A}\right) & \rightarrow\left(X_{M}, F_{t}^{M}\right), \quad \pi_{M}^{B}:\left(X_{B}, F_{t}^{D}\right) \rightarrow\left(X_{M}, F_{t}^{M}\right), \\
& \pi_{M}^{D}:\left(X_{D}, F_{t}^{D}\right) \rightarrow\left(X_{M}, F_{t}^{M}\right) .
\end{aligned}
$$

Corollary 4.6. ([U1, Corollary 5.6]) The flow $\left(X_{M}, F_{t}^{M}\right)$ is determined as the unique maximal common factor flow of those $\left(X_{D}, F_{t}^{D}\right),\left(X_{A}, F_{t}^{A}\right)$, and $\left(X_{B}, F_{t}^{B}\right)$.

This corollary explains all the type classification results mentioned before. Indeed, if $A$ (or $B$ ) is a factor of type $\mathrm{III}_{1}$, then the flow of weights $\left(X_{A}, F_{t}^{A}\right)$ (resp. $\left(X_{B}, F_{t}^{B}\right)$ ) is trivial (see $[\mathrm{CT}]$,[T2]), i.e., the one-point flow, and hence the corollary says that so is the flow of weights $\left(X_{M}, F_{t}^{M}\right)$, which means that $M$ is of type $\mathrm{III}_{1}$. The others can be also explained similarly.

Corollary 4.7. ([U1, Corollary 5.8]) If $A$ and $B$ coincide with each other, i.e., $A=B$, then the amalgamated free product $M=A *_{D} B\left(=A *_{D} A\right)$ and $A(=B)$ have the same flow of weights. In particular, any ergodic flow can be realized as the flow of weights of a certain amalgamated free product.

Remarks 4.8. A few remarks are in order.

(1) Corollary 4.6 has the trivial reformulation: The flow of weights $\left(X_{M}, F_{t}^{M}\right)$ coincides with the associated flow $([\mathrm{HOO}],[\mathrm{Kr}],[\mathrm{FM}])$ of the canonical equivalence relation $\mathcal{R}_{M}$ introduced in $\S 3$.

(2) Based on Theorem 4.4, (4.3) (or (4.5)) together with the proof of Theorem 4.1, we can show the following: Let $A$ and $B$ be von Neumann algebras (with separable preduals) having no type I direct summand, and let $D$ be a common Cartan subalgebra. Then we have

$$
\mathcal{Z}\left(\widetilde{A *_{D} B}\right)=\mathcal{Z}(\widetilde{A}) \cap \mathcal{Z}(\widetilde{B})(\subseteq \widetilde{D})
$$

This implies

$$
\mathcal{Z}\left(A *_{D} B\right)=\mathcal{Z}\left(\widetilde{A *_{D} B}\right)^{\theta^{M}}=\mathcal{Z}(\widetilde{A})^{\theta^{A}} \cap \mathcal{Z}(\widetilde{B})^{\theta^{B}}=\mathcal{Z}(A) \cap \mathcal{Z}(B)(\subseteq D)
$$


thanks to (4.4) and the continuous decomposition theorem [T2].

(3) The (4.8) in the above (2) can be reformulated as follows: Under the assumption that both $A$ and $B$ have no type I direct summand, the amalgamated free product $M=A *_{D} B$ is a factor if and only if the canonical equivalence relation $\mathcal{R}_{M}$ is ergodic.

\section{Miscellaneous Results}

5.1. Let $A \supseteq D \subseteq B$ and $E_{D}^{A}: A \rightarrow D, E_{D}^{B}: B \rightarrow D$ be as in $\S 2$, and let

$$
\left(M, E_{D}^{M}\right)=\left(A, E_{D}^{A}\right) *_{D}\left(B, E_{D}^{B}\right)
$$

be the amalgamated free product. Suppose that $M$ has separable predual, or equivalently that so do both $A$ and $B$, and further that we have known that

$$
\mathcal{Z}(M)=\mathcal{Z}(A) \cap \mathcal{Z}(B) \subseteq \mathcal{Z}(D) .
$$

Then we have the following simultaneous direct integral decompositions:

$$
\begin{aligned}
& M=\int_{\Omega}^{\oplus} M(\omega) d \nu(\omega) \supseteq A=\int_{\Omega}^{\oplus} A(\omega) d \nu(\omega) \supseteq D=\int_{\Omega}^{\oplus} D(\omega) d \nu(\omega) \\
& M=\int_{\Omega}^{\oplus} M(\omega) d \nu(\omega) \supseteq B=\int_{\Omega}^{\oplus} B(\omega) d \nu(\omega) \supseteq D=\int_{\Omega}^{\oplus} D(\omega) d \nu(\omega)
\end{aligned}
$$

with $\mathcal{Z}(M)=L^{\infty}(\Omega, \nu)$. Let

$$
\mathcal{H}=\int_{\Omega}^{\oplus} \mathcal{H}(\omega) d \nu(\omega)
$$

be the corresponding direct integral decomposition of $\mathcal{H}=L^{2}(M)$. We may and do assume that $\omega \mapsto \mathcal{H}(\omega)$ is a constant field of the separable infinite dimensional Hilbert space. The conditional expectations $E_{D}^{M}, E_{D}^{A}, E_{D}^{B}$ are also decomposed as follows:

$$
\begin{gathered}
E_{D}^{M}=\int_{\Omega}^{\oplus}\left(E_{D}^{M}\right)_{\omega} d \nu(\omega), \\
E_{D}^{A}=\int_{\Omega}^{\oplus}\left(E_{D}^{A}\right)_{\omega} d \nu(\omega), \quad E_{D}^{B}=\int_{\Omega}^{\oplus}\left(E_{D}^{B}\right)_{\omega} d \nu(\omega),
\end{gathered}
$$

Theorem 5.1. Under the hypothesis (5.1.1), we have

$$
\left(M(\omega),\left(E_{D}^{M}\right)_{\omega}\right) \cong\left(A(\omega),\left(E_{D}^{A}\right)_{\omega}\right) *_{D(\omega)}\left(B(\omega),\left(E_{D}^{B}\right)_{\omega}\right)
$$

for almost every $\omega \in \Omega$. 
Before going to the proof, we provide a suitable (for our purpose) reformulation of freeness. Let $(N \supseteq L, E: N \rightarrow L)$ be a $L$-probability space, i.e., $N \supseteq L$ is an inclusion of unital algebras with the same unit and $E: N \rightarrow L$ is a conditional expectation in the purely algebraic sense. Assume that $N_{1}, N_{2}$ are unital algebras containing $L$ in common. We introduce the operation $x \in N \mapsto[x]^{\circ}:=x-E(x)$, and the freeness (with amalgamation over $L$ ) of the pair $N_{1}, N_{2}$ can be interpreted as follows:

Lemma 5.2. The pair $N_{1}, N_{2}$ are free with amalgamation over $L$ if and only if the mapping

$$
\Phi_{\left(N_{1}, N_{2}\right)}:\left(x_{1}, x_{2}, \cdots, x_{n}\right) \in \Lambda\left(N_{1}, N_{2}\right) \mapsto E\left(\left[x_{1}\right]^{\circ}\left[x_{2}\right]^{\circ} \cdots\left[x_{n}\right]^{\circ}\right)
$$

is identically zero. Here, $\Lambda\left(N_{1}, N_{2}\right)$ is the set of those finite alternating sequences $\left(x_{1}, x_{2}, \cdots, x_{n}\right)$ of elements in $N_{1} \cup N_{2}$ with $x_{i} \in N_{j(i)}, j(1) \neq j(2) \neq \cdots \neq j(n)$.

In our case, the operation $m \in M \mapsto[m]^{\circ}:=m-E_{D}^{M}(m) \in M^{\circ}:=\operatorname{Ker} E_{D}^{M}$ is normal and linear, and can be (direct integral) decomposed as follows:

$$
[\cdot]^{\circ}=\int_{\Omega}^{\oplus}[\cdot]_{\omega}^{\circ} d \nu(\omega)
$$

where $[m(\omega)]_{\omega}^{\circ}=m(\omega)-\left(E_{D}^{M}\right)_{\omega}(m(\omega))$, a normal linear map, for almost every $\omega \in \Omega$.

Proof. (Proof of Theorem 5.1) Since both $A$ and $B$ have separable preduals, one can choose countable families $\left\{a_{k}\right\}_{k \in \mathbb{N}},\left\{b_{k}\right\}_{k \in \mathbb{N}}$ in such a way that they generate $A$ and $B$, respectively. Let

$$
a_{k}=\int_{\Omega}^{\oplus} a_{k}(\omega) d \nu(\omega), \quad b_{k}=\int_{\Omega}^{\oplus} b_{k}(\omega) d \nu(\omega) .
$$

Then we can choose a co-null Borel subset $\Omega_{1}$ of $\Omega$ in such a way that, for every $\omega \in \Omega_{1}$,

(a) $M(\omega)$ is generated by $A(\omega)$ and $B(\omega)$;

(b) $\left(E_{D}^{M}\right)_{\omega}: M(\omega) \rightarrow D(\omega),\left(E_{D}^{A}\right)_{\omega}: A(\omega) \rightarrow D(\omega),\left(E_{D}^{B}\right)_{\omega}: B(\omega) \rightarrow D(\omega)$ are faithful normal conditional expectations and

$$
\left.\left(E_{D}^{M}\right)_{\omega}\right|_{A(\omega)}=\left(E_{D}^{A}\right)_{\omega},\left.\quad\left(E_{D}^{M}\right)_{\omega}\right|_{B(\omega)}=\left(E_{D}^{B}\right)_{\omega} ;
$$

(c) $A(\omega)$ and $B(\omega)$ are generated by the $a_{k}(\omega)$ 's and the $b_{k}(\omega)$ 's, respectively. Replacing the $a_{k}$ 's (resp. the $b_{k}$ 's) by all the finite products of them and their adjoints from the beginning, we may and do assume, instead of (c), that

(c)' the linear span of the $a_{k}(\omega)$ 's (resp. the $b_{k}(\omega)$ 's) forms a $\sigma$-weakly dense *-subalgebra of $A(\omega)$ (resp. $B(\omega))$. 
The restriction of the map $\Phi_{(A, B)}$ to the subset $\Lambda\left(\left\{a_{k}\right\}_{k \in \mathbb{N}},\left\{b_{k}\right\}_{k \in \mathbb{N}}\right)$ is identically zero by the freeness of the pair $A, B$ (see Lemma 5.2). Therefore, there is a co-null Borel subset $\Omega_{0}$ of $\Omega_{1}$ such that

$$
\begin{aligned}
\Phi_{(A(\omega), B(\omega))} & \left(\left(m_{1}(\omega), m_{2}(\omega), \cdots, m_{n}(\omega)\right)\right) \\
& =\left(E_{D}^{M}\right)_{\omega}\left(\left[m_{1}(\omega)\right]_{\omega}^{\circ}\left[m_{2}(\omega)\right]_{\omega}^{\circ} \cdots\left[m_{n}(\omega)\right]_{\omega}^{\circ}\right)=0
\end{aligned}
$$

for every $\left(m_{1}(x), m_{2}(x), \cdots, m_{n}(x)\right) \in \Lambda\left(\left\{a_{k}(\omega)\right\}_{k \in \mathbb{N}},\left\{b_{k}(\omega)\right\}_{k \in \mathbb{N}}\right)$ and for every $\omega \in \Omega_{0}$. Since the operation $y \mapsto[y]_{\omega}^{\circ}$ is normal and linear, the mapping $\Phi_{(A(\omega), B(\omega))}$ itself is identically zero thanks to (c)' with the aid of the Kaplansky density theorem. Hence, $A(\omega)$ and $B(\omega)$ are free with amalgamation over $D(\omega)$ for every $\omega \in \Omega_{0}$ by Lemma 5.2. Hence we have proved the assertion.

5.2. We would like to apply Theorem 5.1 to amalgamated free products over Cartan subalgebras. In what follows, we suppose that the triple $A \supseteq D \subseteq B$ consists of factors (with separable preduals) of non-type I and a common Cartan subalgebra. The starting point of the discussion is Theorem 4.5, (4.6):

$$
\mathcal{Z}(\widetilde{M})=\mathcal{Z}(\widetilde{A}) \cap \mathcal{Z}(\widetilde{B}) \subseteq \widetilde{D}
$$

Theorem 5.1 implies

Corollary 5.3. Almost every factor $\widetilde{M}(x)$ in the central decomposition

$$
\widetilde{M}=\int_{X_{M}}^{\oplus} \widetilde{M}(x) d \mu(x)
$$

of the continuous core $\widetilde{M}$ can be written as an amalgamated free product over a common Cartan subalgebra.

Here, we further suppose that $M$ is of type $\operatorname{III}_{\lambda}(0<\lambda<1)$, or equivalently that the flow of weights $\left(X_{M}, F_{t}^{M}\right)$ is an (essentially) transitive flow with period $-\log \lambda$ (see [CT],[T2]) so that we may and do assume that

$$
X_{M}=[0,-\log \lambda), \quad F_{t}^{M}=\text { the translation by } t(\bmod :-\log \lambda) .
$$

Since $F_{t}^{M}$ is transitive, we have $\widetilde{M}(0) \cong \widetilde{M}(x)$ for every $x \in X_{M}=[0,-\log \lambda)$, and hence we may and do assume that $x \in X_{M} \mapsto \widetilde{M}(x)$ is a constant field of the type $\mathrm{II}_{\infty}$ factor $\widetilde{M}(0)$. Hence, we conclude

Corollary 5.4. In the current setting, the (unique) type $\mathrm{II}_{\infty}$ factor appearing in the discrete decomposition $([\mathrm{C} 1])$ of $M$ is written as an amalgamated free product over a common Cartan subalgebra. 
5.3. We keep the setting and the notations as in $\S \S 5.2$. It is known that an ergodic free action of a non-amenable discrete group may or may not be amenable ([Z], see also §6), or equivalently the associated von Neumann factor may or may not be injective (see [C2]) (or hyperfinite), and hence it is somewhat non-trivial whether or not the amalgamated free product $M=A *_{D} B$ is non-injective.

Theorem 5.5. In the current setting, there is a copy of the free group factor $L\left(\mathbb{F}_{2}\right)$ in the continuous core $\widetilde{M}$ which is the range of a faithful normal conditional expectation. In particular, $M$ is not injective.

The non-injectivity result follows also from a result in our resent work [U2], where we have shown that the amalgamated free product $M=A *_{D} B$ is not a McDuff factor (under the assumption that both $A$ and $B$ are factors of non-type I). However, the proof below is still valid even in the case that both $A$ and $B$ have no type I direct summand. (See Remarks 4.8, (2),(3).)

Proof. Let $\operatorname{Tr}_{\widetilde{M}}, \operatorname{Tr}_{\widetilde{A}}, \operatorname{Tr}_{\widetilde{B}}, \operatorname{Tr}_{\widetilde{D}}$ be the canonical traces on $\widetilde{M}, \widetilde{A}, \widetilde{B}, \widetilde{D}$, respectively, (scaled in the usual way under the dual actions). It can be checked that

$$
\operatorname{Tr}_{\widetilde{M}}=\operatorname{Tr}_{\widetilde{D}} \circ \widehat{E_{D}^{M}}, \quad \operatorname{Tr}_{\widetilde{A}}=\operatorname{Tr}_{\widetilde{D}} \circ \widehat{E_{D}^{A}}, \quad \operatorname{Tr}_{\widetilde{B}}=\operatorname{Tr}_{\widetilde{D}} \circ \widehat{E_{D}^{B}},
$$

where $\widehat{E_{D}^{M}}: \widetilde{M} \rightarrow \widetilde{D}, \widehat{E_{D}^{A}}: \widetilde{A} \rightarrow \widetilde{D}, \widehat{E_{D}^{B}}: \widetilde{B} \rightarrow \widetilde{D}$ are as in $\S 4$. As in $\S \S 6.1$, we consider the simultaneous direct integral decompositions of the inclusions $\widetilde{M} \supseteq \widetilde{A}, \widetilde{B} \supseteq \widetilde{D}$ and the conditional expectations $\widehat{E_{D}^{M}}: \widetilde{M} \rightarrow \widetilde{D}, \widehat{E_{D}^{A}}: \widetilde{A} \rightarrow \widetilde{D}$, $\widehat{E_{D}^{B}}: \widetilde{B} \rightarrow \widetilde{D}$ subject to the central decomposition

$$
\widetilde{M}=\int_{X_{M}}^{\oplus} \widetilde{M}(x) d \mu(x)
$$

thanks to Theorem 4.5, (4.6), and let

$$
\operatorname{Tr}_{\widetilde{D}}=\int_{X_{M}}^{\oplus}\left(\operatorname{Tr}_{\widetilde{D}}\right)_{x} d \mu(x)
$$

be the corresponding direct integral decomposition.

Note that the continuous core $\widetilde{M}$ is of type $\mathrm{II}_{\infty}$ or of type $\mathrm{II}_{1}$. (Recall that, the continuous core of a von Neumann algebra of type III must be of type $\mathrm{II}_{\infty}$, while there is no type change for the other types.) We assume that $\widetilde{M}$ is of type $\mathrm{II}_{\infty}$ in what follows, since the quite similar (actually simpler) argument as below apparently works in the type $\mathrm{II}_{1}$ case.

Let $X$ be a co-null Borel subset of $X_{M}$ satisfying the same conditions (a), (b), (c) as in the proof of Theorem 5.1 with the additional ones: 
(e) Both $\widetilde{A}(x)$ and $\widetilde{B}(x)$ are von Neumann algebras of type II for every $x \in X$. (This follows from the assumption that $A$ and $B$ have no type I direct summand.)

(f) $\left(\operatorname{Tr}_{\widetilde{D}}\right)_{x} \circ\left(\widehat{E_{D}^{M}}\right)_{x}$ is a faithful normal semi-finite trace, and thus so are $\operatorname{both}\left(\operatorname{Tr}_{\widetilde{D}}\right)_{x} \circ\left(\widehat{E_{D}^{A}}\right)_{x},\left(\operatorname{Tr}_{\widetilde{D}}\right)_{x} \circ\left(\widehat{E_{D}^{B}}\right)_{x}$.

By choosing a much smaller co-null subset instead of $X$ if necessary, the set of those $(x, p) \in X \times B\left(\mathcal{H}_{0}\right)$ with the separable Hilbert space $\mathcal{H}_{0}$ (on which almost every $\widetilde{D}(\omega)$ act), satisfying

- $p=p^{2}=p^{*} \in \widetilde{D}(x)$;

- $\left(\operatorname{Tr}_{\widetilde{D}}\right)_{x}(p)=1$

can be assumed to be Borel. Therefore, the measurable selection principle enables us to choose a measurable field $x \in X \mapsto p_{x} \in \widetilde{D}(x)$ of projections such that $\left(\operatorname{Tr}_{\widetilde{D}}\right)_{x}\left(p_{x}\right)=1$ for every $x \in X$, and we set

$$
p:=\int_{X_{M}}^{\oplus} p_{x} d \mu(x)
$$

Since $\widetilde{M}$ is of type $\mathrm{II}_{\infty}$, we have

$$
\begin{aligned}
\int_{X_{M}}^{\oplus} \widetilde{M}(x) d \mu(x) & =\widetilde{M}=(p \widetilde{M} p) \otimes B\left(\mathcal{H}_{0}\right) \\
& =\int_{X_{M}}^{\oplus}\left(p_{x} \widetilde{M}(x) p_{x}\right) \otimes B\left(\mathcal{H}_{0}\right) d \mu(x)
\end{aligned}
$$

with the separable Hilbert space $\mathcal{H}_{0}$. Then we see that, for every $x \in X$,

- both $p_{x} \widetilde{A}(x) p_{x}$ and $p_{x} \widetilde{B}(x) p_{x}$ are of type $\mathrm{II}_{1}$ (thanks to (e),(f));

- $p_{x} \widetilde{A}(x) p_{x}, p_{x} \widetilde{B}(x) p_{x}$ are free with amalgamation over $\widetilde{D}(x) p_{x}$ in the $\widetilde{D}(x) p_{x^{-}}$ probability space $\left(p_{x} \widetilde{M}(x) p_{x},\left.\left(\widehat{E_{D}^{M}}\right)_{x}\right|_{p_{x} \widetilde{M}(x) p_{x}}\right)$ (by (b), (c) and the proof of Theorem 5.1).

Repeating the argument of the type $\mathrm{III}_{0}$ case in the proof of [U1, Lemma 4.2], we choose two measurable fields $x \in X \mapsto u_{x} \in p_{x} \widetilde{A}(x) p_{x}, x \in X \mapsto v_{x} \in p_{x} \widetilde{B}(x) p_{x}$ of unitaries satisfying

$$
\left(\widehat{E_{D}^{A}}\right)_{x}\left(\left(u_{x}\right)^{n}\right)=0, \quad\left(\widehat{E_{D}^{B}}\right)_{x}\left(\left(v_{x}\right)^{n}\right)=0
$$

for every $n(\neq 0) \in \mathbb{Z}$, and set

$$
u:=\int_{X_{M}}^{\oplus} u_{x} d \mu(x), \quad v:=\int_{X_{M}}^{\oplus} v_{x} d \mu(x) .
$$


Then $u, v$ are free Haar unitaries, and hence the von Neumann subalgebra $N:=$ $\{u, v\}^{\prime \prime}$ of $p \widetilde{M} p$ is isomorphic to the free group factor $L\left(\mathbb{F}_{2}\right)$. Notice that $N$ is decomposable relative to $\mathcal{Z}(\widetilde{M})=L^{\infty}\left(X_{M}, \mu\right)$ and that $u_{x}, v_{x}$ also are free Haar unitaries for almost every $x \in X_{M}$. Therefore, we have

$$
L\left(\mathbb{F}_{2}\right) \cong N(x)=\left\{u_{x}, v_{x}\right\}^{\prime \prime} \subseteq p_{x} \widetilde{M}(x) p_{x} \text { for almost every } x \in X_{M},
$$

and thus

$$
\begin{aligned}
L\left(\mathbb{F}_{2}\right) \otimes B\left(\mathcal{H}_{0}\right) \otimes \mathbf{C} 1 & \subseteq L\left(\mathbb{F}_{2}\right) \otimes B\left(\mathcal{H}_{0}\right) \otimes \mathcal{Z}(\widetilde{M}) \\
& =\int_{X_{M}}^{\oplus} L\left(\mathbb{F}_{2}\right) \otimes B\left(\mathcal{H}_{0}\right) d \mu(x) \\
& \subseteq \int_{X_{M}}^{\oplus}\left(p_{x} \widetilde{M}(x) p_{x}\right) \otimes B(\mathcal{H}) d \mu(x) \\
& =\int_{X_{M}}^{\oplus} \widetilde{M}(x) d \mu(x) .
\end{aligned}
$$

Since $p \widetilde{M} p$ is of type $\mathrm{II}_{1}$, the copy of $L\left(\mathbb{F}_{2}\right) \otimes B\left(\mathcal{H}_{0}\right)$ in $\widetilde{M}$ (or in $\widetilde{M}(x)$ for almost every $x \in X_{M}$ ) is clearly the range of a faithful normal conditional expectation. Hence we are done.

Since the copy of $L\left(\mathbb{F}_{2}\right)$ constructed in the proof is well-behaved with the central decomposition of $\widetilde{M}$, we have

Corollary 5.6. Keep the same setting as in Theorem 5.5. If the amalgamated free product $M=A *_{D} B$ is of type $\mathrm{III}_{\lambda}$, then the type $\mathrm{II}_{\infty}$ factor appearing in the discrete decomposition of $M$ contains a copy of the free group factor $L\left(\mathbb{F}_{2}\right)$ which is the range of a faithful normal conditional expectation. Therefore, so does the $M$ itself.

Proof. The first part of the assertion is clear from the proof of Theorem 5.5 together with Corollary 5.4. The latter follows from the first half and the discrete decomposition theorem $([\mathrm{C} 1])$ for type $\mathrm{III}_{\lambda}$ factors.

For a while, we assume that both $A$ and $B$ are general factors (not necessary of non-type I) and that $D$ is a common Cartan subalgebra. If either $A$ or $B$ is of type $\mathrm{I}_{n}$ with possibly $n=\infty$, then both must coincide, i.e., $A=B=M_{n}(\mathbf{C})$ or $B(\mathcal{H})$. In this case, we can see that $M=A *_{D} B\left(=A *_{D} A\right)$ is isomorphic to $L\left(\mathbb{F}_{n-1}\right) \otimes A$. Therefore, we obtain

Corollary 5.7. The amalgamated free product $M=A *_{D} B$ of factors (with separable preduals) over a common Cartan subalgebra is injective if and only if either $A$ or $B$ is of type $\mathrm{I}_{2}$ (and hence $A=B=M_{2}(\mathbf{C})$ and $D$ is the diagonals). 
This corollary can be thought of as an analogue of the following classical group theoretical fact: A free product group $G * H$ is amenable if and only if $G=H=$ $\mathbb{Z}_{2}$.

5.4. We keep the same setting and the notations as in $\S \S 5.2$ even in this subsection. We would like here to show that the amalgamated free product $M=A *_{D} B$ is not related to any free group factor as a simple application of the striking result [V2] of D. Voiculescu with the aid of Theorem 5.1 (or Corollary 5.3, Corollary 5.4). A similar application of Voiculescu's result was also given by D. Shlyakhtenko [S1] in a different context.

When the amalgamated free product $M=A *_{D} B$ is of type $\mathrm{II}_{1}$, we can apply directly Voiculescu's theorem to the case since the normalizer $\mathcal{N}_{M}(D)$ generates the whole $M$, and hence $M$ is not isomorphic to any (interpolated) free group factor $L\left(\mathbb{F}_{r}\right)$ with $0<r \leq \infty$. Thus it suffices to consider only the infinite cases, and we start with the following lemma:

Lemma 5.8. Let $N \supseteq C$ be a factor of type $\mathrm{II}_{\infty}$ and an abelian von Neumann subalgebra. Let $\operatorname{Tr}$ be a faithful normal semi-finite trace on $N$ such that $\left.\operatorname{Tr}\right|_{C}$ is semi-finite. Suppose that the normalizer $\mathcal{N}_{N}(C)$ generates the whole $N$. Then, for each finite (in $N$ ) non-zero projection $p \in C$ (such a projection indeed exists since $\left.\operatorname{Tr}\right|_{C}$ is semi-finite), the normalizer $\mathcal{N}_{p N p}(C p)$ generates the whole $p N p$.

Proof. By assumption, we see that the linear span of $\mathcal{N}_{N}(C)$ forms a $\sigma$-weakly dense $*$-subalgebra in $N$. Hence, the linear span of elements of the form pup with $u \in \mathcal{N}_{N}(C)$ also is $\sigma$-weakly dense in $p N p$. Since $p$ is finite in $N, p N p$ is a factor of type $I I_{1}$. Let us denote $q$ and $r$ the support and the range projections of pup, respectively. Then the projections $q, r$ are in $p N p$ since $q, r \leq p$. We here need the following fact:

Fact 5.9. If $p \neq q$ (or equivalently $p \neq r$ thanks to the fact that $p N p$ is finite), then there is an element $w \in \mathcal{G N}_{p N p}(C p)$ with $w^{*} w=p-q$, ww $w^{*}=p-r$. Here, $\mathcal{G N}_{p N p}(C p)$ denotes the normalizing groupoid, i.e., the set of those partial isometries $v \in N$ such that $v^{*} v, v v^{*} \in C$ and $v C v^{*}=C v v^{*}, v^{*} C v=C v^{*} v$.

Proof. (Proof of the Fact.) Since $N$ is a factor, we have $(p-r) N(p-q) \neq\{0\}$, and hence there is a unitary $v \in \mathcal{N}_{N}(C)$ such that $(p-r) v(p-q)$ is not equal zero. Thus, there is a non-zero element $v_{0} \in \mathcal{G N}_{p N p}(C p)$ such that $v_{0}^{*} v_{0} \leq p-q$ and $v_{0} v_{0}^{*} \leq p-r$. We can do the standard exhaustion argument thanks to the fact that $p N p$ is finite. Hence we get a desired partial isometry.

Let $w:=$ pup with $u \in \mathcal{N}_{N}(C)$, and the above fact says that we can choose a unitary $\widetilde{w} \in \mathcal{N}_{p N p}(C p)$ in such a way that $w=r \widetilde{w} q$. Moreover, $q, r$ can be 
written as finite linear combinations of unitries in $C p$, and hence $r \widetilde{w} q$ is a finite linear combinations of elements in $\mathcal{N}_{p N p}(C p)$. Therefore, any element in $p N p$ can be approximated $\sigma$-weakly by finite linear combinations of elements in $\mathcal{N}_{p N p}(C p)$. Hence we complete the proof of the lemma.

Proposition 5.10. Keep the same setting as in Theorem 5.5.

(1) If the amalgamated free product $M=A *_{D} B$ is of type $\mathrm{II}_{\infty}$, then $M$ is not isomorphic to any $L\left(\mathbb{F}_{r}\right) \otimes B(\mathcal{H})$ with $0<r \leq \infty$.

(2) If the amalgamated free product $M=A *_{D} B$ is of type III, then almost every

type $\mathrm{II}_{\infty}$ factor appearing in the central decomposition of the continuous core $\widetilde{M}$ is not isomorphic to any $L\left(\mathbb{F}_{r}\right) \otimes B(\mathcal{H})$ with $0<r \leq \infty$.

(3) If the amalgamated free product $M=A *_{D} B$ is of type $\mathrm{III}_{\lambda}(0<\lambda<1)$, then

the type $\mathrm{II}_{\infty}$ factor appearing in the discrete decomposition is not isomorphic to any $L\left(\mathbb{F}_{r}\right) \otimes B(\mathcal{H})$ with $0<r \leq \infty$.

Proof. All the assertions follow from [V2, 5,3 Theorem, 7.4 Corollary] with the aid of Lemma 5.8. When showing the assertions (2), (3), we further need Corollary 5.3, Corollary 5.4, respectively.

\section{Example I. Boundary Actions of Free Groups}

6.1. Let $\mathfrak{X}$ be a finite set with $|\mathfrak{X}| \geq 2$, and we set $\mathfrak{X}^{-1}:=\left\{x^{-1} ; x \in \mathfrak{X}\right\}$. We consider the free group $\Gamma:=\mathbb{F}(\mathfrak{X})$ over the generators $\mathfrak{X}$ and its boundary $\partial \Gamma$. In this case, the boundary $\partial \Gamma$ is defined as the one-sided shift space of the alphabets $\mathfrak{X} \cup \mathfrak{X}^{-1}$ determined by the forbidden blocks $\left(x^{-1} x\right),\left(x x^{-1}\right)$, equipped with the usual product topology. It is plain to see that $\partial \Gamma$ is identified with the set of semi-infinite reduced words in $\mathfrak{X} \cup \mathfrak{X}^{-1}$. We will freely use these two different descriptions in what follows. The group $\Gamma$ acts topologically on the boundary $\partial \Gamma$ by the left multiplication, i.e., for $\gamma \in \Gamma$ and for $\omega=\omega_{1} \omega_{2} \cdots \in \partial \Gamma$,

$$
\gamma \cdot \omega:=\text { the reduced form of the word } \gamma \omega_{1} \omega_{2} \cdots .
$$

6.2. We decompose the set $\mathfrak{X}$ into two disjoint non-empty subsets $\mathfrak{X}_{1}, \mathfrak{X}_{2}$ with $\mathfrak{X}=\mathfrak{X}_{1} \sqcup \mathfrak{X}_{2}$. Then we have $\Gamma=\Gamma_{1} * \Gamma_{2}$ with $\Gamma_{1}=\mathbb{F}\left(\mathfrak{X}_{1}\right), \Gamma_{2}=\mathbb{F}\left(\mathfrak{X}_{2}\right)$. The boundaries $\partial \Gamma_{1}, \partial \Gamma_{2}$ can be (topologically) embedded into $\partial \Gamma$ as follows:

$$
\begin{aligned}
\partial \Gamma_{1}= & \text { the shift subspace of } \partial \Gamma \\
& \text { with extra forbidden blocks }(x), x \in \mathfrak{X}_{2}, \\
\partial \Gamma_{2}= & \text { the subset of } \partial \Gamma \\
& \text { with extra forbidden blocks }(x), x \in \mathfrak{X}_{1} .
\end{aligned}
$$


Therefore, the subspaces $\partial \Gamma_{1}, \partial \Gamma_{2}$ are closed and invariant under the actions of $\Gamma_{1}, \Gamma_{2}$, respectively. We consider the following disjoint decompositions:

$$
\partial \Gamma=\left(\partial \Gamma_{1}\right)^{c} \sqcup \partial \Gamma_{1}, \quad \partial \Gamma=\left(\partial \Gamma_{2}\right)^{c} \sqcup \partial \Gamma_{2},
$$

and note that $\left(\partial \Gamma_{1}\right)^{c},\left(\partial \Gamma_{2}\right)^{c}$ are open and invariant under the actions of $\Gamma_{1}, \Gamma_{2}$, respectively. We define

$$
\begin{aligned}
& \left(\partial \Gamma_{1}\right)^{\perp}:=\left\{\omega=\left(\omega_{n}\right)_{n=1}^{\infty} \in(\partial \Gamma)^{c} ; \omega_{1} \in \mathfrak{X}_{2} \cup\left(\mathfrak{X}_{2}\right)^{-1}\right\}, \\
& \left(\partial \Gamma_{2}\right)^{\perp}:=\left\{\omega=\left(\omega_{n}\right)_{n=1}^{\infty} \in(\partial \Gamma)^{c} ; \omega_{1} \in \mathfrak{X}_{1} \cup\left(\mathfrak{X}_{1}\right)^{-1}\right\} .
\end{aligned}
$$

Let us define the map $\Phi_{1}: \Gamma_{1} \times\left(\partial \Gamma_{1}\right)^{\perp} \rightarrow\left(\partial \Gamma_{1}\right)^{c}$ as the restriction of the action $\operatorname{map} \Gamma \times \partial \Gamma \rightarrow \partial \Gamma$, i.e.,

$$
\Phi_{1}:\left\{\begin{array}{l}
(e, \omega) \mapsto \omega \\
\left(\gamma_{1} \cdots \gamma_{2},\left(\omega_{n}\right)_{n=1}^{\infty}\right) \mapsto\left(\gamma_{1}, \ldots, \gamma_{n}, \omega_{1}, \omega_{2}, \ldots\right)
\end{array}\right.
$$

for each reduced word $\gamma_{1} \cdots \gamma_{n}$. Similarly, the map $\Phi_{2}: \Gamma_{2} \times\left(\partial \Gamma_{2}\right)^{\perp} \rightarrow\left(\partial \Gamma_{2}\right)^{c}$ is defined as the restriction of the action map $\Gamma \times \partial \Gamma \rightarrow \partial \Gamma$. We here note that the topology on $\partial \Gamma$ is generated by the family of clopen sets of the form:

$$
\Omega(\gamma)=\left\{\omega=\left(\omega_{n}\right)_{n=1}^{\infty} \in \partial \Gamma ; \omega_{1}=\gamma_{1}, \ldots, \omega_{n}=\gamma_{n}\right\}
$$

with a reduced word $\gamma=\gamma_{1} \cdots \gamma_{n}$.

Lemma 6.1. We have

$$
\begin{aligned}
& \left(\partial \Gamma_{1}\right)^{c}=\bigsqcup_{\gamma \in \Gamma_{1}} \bigsqcup_{\omega \in \mathfrak{X}_{2} \cup\left(\mathfrak{X}_{2}\right)^{-1}} \Omega(\gamma \omega), \\
& \left(\partial \Gamma_{1}\right)^{c}=\bigsqcup_{\gamma \in \Gamma_{2}} \bigsqcup_{\omega \in \mathfrak{X}_{1} \cup\left(\mathfrak{X}_{1}\right)^{-1}} \Omega(\gamma \omega) .
\end{aligned}
$$

It is plain to check that

(1) the maps $\Phi_{1}, \Phi_{2}$ are bijections;

(2) $\Phi_{k}\left(\{\gamma\} \times \Omega\left(\omega_{1} \cdots \omega_{n}\right)\right)=\Omega\left(\gamma \omega_{1} \cdots \omega_{n}\right)(k=1,2)$

with a reduced word $\omega_{1} \cdots \omega_{n}$.

Therefore, thanks to Lemma 6.1 , we see that $\Phi_{1}, \Phi_{2}$ send all the basic clopen sets to all those, when the product topologies of the discrete one and the induced one from $\partial \Gamma$ are considered on both $\Gamma_{1} \times\left(\partial \Gamma_{1}\right)^{\perp}, \Gamma_{2} \times\left(\partial \Gamma_{2}\right)^{\perp}$. It is also plain to check that

$$
\Phi_{k}\left(\gamma_{1} \gamma_{2}, \omega\right)=\gamma_{1} \cdot \Phi_{k}\left(\gamma_{2}, \omega\right) \quad(k=1,2),
$$

and hence we conclude 
Proposition 6.2. The maps $\Phi_{1}: \Gamma_{1} \times\left(\partial \Gamma_{1}\right)^{\perp} \rightarrow\left(\partial \Gamma_{1}\right)^{c}, \Phi_{2}: \Gamma_{2} \times\left(\partial \Gamma_{2}\right)^{\perp} \rightarrow$ $\left(\partial \Gamma_{2}\right)^{c}$ are homeomorphisms, and via these homeomorphisms, the actions of $\Gamma_{1}$, $\Gamma_{2}$ on $\left(\partial \Gamma_{1}\right)^{c},\left(\partial \Gamma_{2}\right)^{c}$ are conjugate to those of $\Gamma_{1}, \Gamma_{2}$ on $\Gamma_{1} \times\left(\partial \Gamma_{1}\right)^{\perp}, \Gamma_{1} \times\left(\partial \Gamma_{2}\right)^{\perp}$ which are defined as the product action of the translation and the trivial one.

6.3. Let $n=|\mathfrak{X}|$ and $n_{1}=\left|\mathfrak{X}_{1}\right|, n_{2}=\left|\mathfrak{X}_{2}\right|$. We discuss here the probability measure $\mu$ on the boundary $\partial \Gamma$ defined in such a way that

$$
\mu(\Omega(\gamma)):=\frac{1}{2 n}\left(\frac{1}{2 n-1}\right)^{\ell(\gamma)-1}
$$

with the word length function $\ell(\cdot)$. It is known that the measure $\mu$ is quasiinvariant under the action of $\Gamma$. (See $[\mathrm{KS}]$.) The non-singular action of $\Gamma$ on the probability space $(\partial \Gamma, \mu)$ can be checked to be free and ergodic (see [RR], [KS],[PS]). Moreover, J. Ramagge \& G. Robertson [RR] showed that the action of $\Gamma$ is of type III $\frac{1}{2 n-1}$ so that the crossed-product $M=L^{\infty}(\partial \Gamma, \mu) \rtimes \Gamma$ is a factor of type III $\frac{1}{2 n-1}$. Moreover, S. Adams' result [A] (see also [Ver, Example 2 in p $\dot{8} 9$ ]) implies that the factor is injective. (It should be remarked that the Cuntz-Krieger algebra interpretation for boundary actions provided by J. Spielberg [Sp] together with M. Enomoto, M. Fujii \& Y. Watatani [EFW] also shows that the crossed-product is the injective factor of type III $\frac{1}{2 n-1}$.) Set $A:=L^{\infty}(\partial \Gamma, \mu) \rtimes \Gamma_{1}$, $B:=L^{\infty}(\partial \Gamma, \mu) \rtimes \Gamma_{2}$, and it is plain to see that, the crossed-product is written as an amalgamated free product over a common Cartan subalgebra, that is, we have $M \cong A *_{D} B$ with $D:=L^{\infty}(\partial \Gamma, \mu)$. Therefore, the boundary action of the free group $\Gamma$ provides an example of an injective factor arising as an amalgamated free product over a common Cartan subalgebra.

6.4. Since

$$
\left|\left\{\gamma \in \Gamma_{k} ; \ell(\gamma)=m\right\}\right|=2 n_{k}\left(2 n_{k}-1\right)^{m-1} \quad(k=1,2),
$$


we have

$$
\begin{aligned}
& \mu\left(\left(\partial \Gamma_{1}\right)^{c}\right)=\mu\left(\bigsqcup_{\gamma \in \Gamma_{1}} \bigsqcup_{\omega \in \mathfrak{X}_{2} \cup\left(\mathfrak{X}_{2}\right)^{-1}} \Omega(\gamma \omega)\right) \quad(\text { Lemma 6.1) } \\
& =\sum_{\omega \in \mathfrak{X}_{2} \cup\left(\mathfrak{X}_{2}\right)^{-1}} \mu(\Omega(\omega))+\sum_{m=1}^{\infty} \sum_{\substack{\gamma \in \Gamma_{1} \\
\ell(\gamma)=m}} \sum_{\omega \in \mathfrak{X}_{2} \cup\left(\mathfrak{X}_{2}\right)^{-1}} \mu(\Omega(\gamma \omega)) \\
& =2 n_{2} \frac{1}{2 n}+\sum_{m=1}^{\infty}\left(2 n_{1}\left(2 n_{1}-1\right)^{m-1}\right) 2 n_{2}\left(\frac{1}{2 n}\left(\frac{1}{2 n-1}\right)^{m}\right) \\
& =\frac{n_{2}}{n}+\frac{2 n_{1} n_{2}}{n(2 n-1)} \cdot \sum_{m=1}^{\infty}\left(\frac{2 n_{1}-1}{2 n-1}\right)^{m-1} \\
& =1 \quad\left(\text { by } n=n_{1}+n_{2}\right) .
\end{aligned}
$$

Similarly, we have $\mu\left(\left(\partial \Gamma_{2}\right)^{c}\right)=1$. Hence, we obtain

$$
\mu\left(\partial \Gamma_{1}\right)=\mu\left(\partial \Gamma_{2}\right)=0 .
$$

Furthermore, we have

$$
\begin{array}{r}
\mu\left(\Phi_{k}\left(\{\gamma\} \times \Omega\left(\omega_{1} \cdots \omega_{m}\right)\right)\right)=\frac{1}{2 n}\left(\frac{1}{2 n-1}\right)^{\ell(\gamma)+m-1} \\
=\left(\frac{1}{2 n-1}\right)^{\ell(\gamma)} \times \frac{1}{2 n}\left(\frac{1}{2 n-1}\right)^{m-1}
\end{array}
$$

and hence

$$
\left(\left.\mu\right|_{\left(\partial \Gamma_{k}\right)^{c}}\right) \circ \Phi_{k}=\delta_{k} \otimes\left(\left.\mu\right|_{\left(\partial \Gamma_{k}\right)^{\perp}}\right) \quad(k=1,2)
$$

with the measure $\delta_{k}(\{\gamma\})=\left(\frac{1}{2 n-1}\right)^{\ell(\gamma)}$ equivalent to the counting measure. From the discussions above, we conclude

Proposition 6.3. We have

$$
\begin{aligned}
& L^{\infty}(\partial \Gamma, \mu) \rtimes \Gamma_{1}=L^{\infty}\left(\left(\partial \Gamma_{1}\right)^{c}, \mu\right) \rtimes \Gamma_{1} \\
& \cong\left(\ell^{\infty}\left(\Gamma_{1}\right) \rtimes \Gamma_{1}\right) \otimes L^{\infty}\left(\left(\partial \Gamma_{1}\right)^{\perp},\left.\mu\right|_{\left(\partial \Gamma_{1}\right)^{\perp}}\right), \\
& L^{\infty}(\partial \Gamma, \mu) \rtimes \Gamma_{2}=L^{\infty}\left(\left(\partial \Gamma_{2}\right)^{c}, \mu\right) \rtimes \Gamma_{2} \\
& \cong\left(\ell^{\infty}\left(\Gamma_{2}\right) \rtimes \Gamma_{2}\right) \otimes L^{\infty}\left(\left(\partial \Gamma_{2}\right)^{\perp},\left.\mu\right|_{\left(\partial \Gamma_{2}\right)^{\perp}}\right) .
\end{aligned}
$$

The isomorphisms are induced from the maps $\Phi_{1}, \Phi_{2}$, respectively. In particular, the crossed-products both are of homogeneous type $\mathrm{I}_{\infty}$. 
Therefore, we have seen that the free components of our injective amalgamated free product $M=A *_{D} B$ both are of homogeneous type $\mathrm{I}_{\infty}$.

6.5. At the end of this section, we give a criterion on injectivity of amalgamated free products over Cartan subalgebras. Let $M=A *_{D} B$ be an amalgamated free product over a common Cartan subalgebra. Here, we do not assume that $A$ and $B$ are factors nor that they have no type I direct summand. We choose central projections $p_{A}, p_{B}$ of $A, B$ in such a way that both $A p_{A}$ and $B p_{B}$ have no type I direct summand. Since $D$ is a Cartan subalgebra in both $A$ and $B$, the projections $p_{A}, p_{B}$ are in $D$ so that $p:=p_{A} p_{B}=p_{B} p_{A}$ is also a projection in $D$. Suppose here that $p$ is non-zero. Then the reduced von Neumann algebra $p M p$ contains both $p A p$ and $p B p$, and their freeness can be easily checked with respect to the conditional expectation

$$
\left(E_{D}^{M}\right)_{p}:=\left.E_{D}^{M}\right|_{p M p}: p M p \rightarrow D p .
$$

It can be easily checked that the continuous cores satisfy

$$
\widetilde{p M p}=p \widetilde{M} p, \quad \widetilde{p A p}=p \widetilde{A} p, \quad \widetilde{p B p}=p \widetilde{B} p, \quad \widetilde{D p}=\widetilde{D} p .
$$

Hence we get the inclusion relations

$$
\widetilde{p M p} \supseteq \widetilde{p A p} \supseteq \widetilde{D p}, \quad \widetilde{p M p} \supseteq \widetilde{p B p} \supseteq \widetilde{D p} .
$$

We can easily see that the conditional expectation

$$
\widetilde{\left(E_{D}^{M}\right)_{p}}: \widetilde{p M p} \rightarrow \widetilde{D p}
$$

coincides with

$$
\left(\widetilde{E_{D}^{M}}\right)_{p}:=\left.\widetilde{E_{D}^{M}}\right|_{p \widetilde{M} p}: p \widetilde{M} p \rightarrow \widetilde{D} p .
$$

Hence we can show that, the von Neumann subalgebra

$$
N:=\widetilde{p A p} \vee \widetilde{p B p}(\subseteq \widetilde{p M p})
$$

is identified with the amalgamated free product over a common Cartan subalgebra

$$
(\widetilde{p A p}) * \widetilde{D p}(\widetilde{p B p}) \text {. }
$$

Notice here that

- both $p A p$ and $p B p$ have no type I direct summand;

- there is a faithful normal conditional expectation from $\widetilde{p M p}$ onto $N$ since $N$ is invariant under the modular action $\sigma_{t}^{\psi / \widetilde{\left.E_{D}^{M}\right)_{p}}}(t \in \mathbf{R})$ with a faithful normal state $\psi$ on $\widetilde{D p}$ thanks to [T1] and Theorem 1.1 . 
Thus we apply the same argument as in the proof of Theorem 5.5 (see after the statement of that theorem) to the amalgamated free product $N$, and as a consequence we get a copy of the free group factor in $\widetilde{p M p}$ as the range of a faithful normal conditional expectation from $N$ (and hence from $\widetilde{p M p}$ ). Therefore, $p M p$ is not injective, and neither is $M$. Therefore, we conclude

Proposition 6.4. In the current setting, if the amalgamated free product $M=$ $A *_{D} B$ is injective, then the non-type $I$ direct summands in $A$ and $B$ need not meet (in D), i.e., their support central projections are disjoint (in D).

Remark 6.5. One can construct two von Neumann algebras $A, B$ with a common Cartan subalgebra $D$ in such a way that (i) $A$ has the non-type I direct summand and $B \cong L^{\infty}(\Omega) \otimes B(\mathcal{H})$ (possibly with any dimension $\operatorname{dim} \mathcal{H} \geq 2$ ); (ii) the amalgamated free product $A *_{D} B$ is injective. (Compare with Proposition 6.3, 6.4.)

\section{Example II. Number of Free Components}

Let $A \supseteq D \subseteq B$ be $\sigma$-finite von Neumann algebras with faithful normal conditional expectations $E_{D}^{A}: A \rightarrow D, E_{D}^{B}: B \rightarrow D$, which are assumed to be of the form:

$$
\begin{gathered}
A=A_{0} \otimes B\left(\ell^{2}(\mathbb{N})\right), \quad B=B_{0} \otimes B\left(\ell^{2}(\mathbb{N})\right), \quad D=D_{0} \otimes \ell^{\infty}(\mathbb{N}), \\
E_{D}^{A}\left(a \otimes e_{i j}\right)=\delta_{i j} E_{D_{0}}^{A_{0}}(a), \quad E_{D}^{A}\left(b \otimes e_{i j}\right)=\delta_{i j} E_{D_{0}}^{B_{0}}(b)
\end{gathered}
$$

with

$$
B\left(\ell^{2}(\mathbb{N})\right)=\left\{e_{i j}\right\}^{\prime \prime} \supseteq \ell^{\infty}(\mathbb{N})=\left\{e_{i i}\right\}^{\prime \prime},
$$

where the $e_{i j}$ 's are the natural matrix units and will be denoted by $e_{i j}^{A}$ or $e_{i j}^{B}$ instead of $e_{i j}$ when regarded as elements in $A$ or $B$, to avoid any confusion. We further suppose that $B_{0}$ (and hence $B$ itself) is injective or hyperfinite, has no type I direct summand, and that $D_{0}$ is a Cartan subalgebra. Thanks to A. Connes, J. Feldman \& B. Weiss [CFW], we may and do assume that there is a unitary $u \in B_{0}$ such that

$$
B_{0}=<D_{0}, u>^{\prime \prime}, \quad E_{D_{0}}^{B_{0}}\left(u^{n}\right)=0 \text { as long as } n \neq 0,
$$

and the automorphism $\operatorname{Ad} u \in \operatorname{Aut}\left(D_{0}\right)$ is denoted by $\alpha$.

In this setting, we will investigate the reduced von Neumann algebra $p M p$ of the amalgamated free product:

$$
\left(M, E_{D}^{M}\right)=\left(A, E_{D}^{A}\right) *_{D}\left(B, E_{D}^{B}\right)
$$

by a minimal projection $p:=1 \otimes e_{11}$ in the common subalgebra $\mathbf{C} 1 \otimes \ell^{\infty}(\mathbb{N})$. 
We introduce the following notation rule:

$$
[a]_{i j}^{A}:=a \otimes e_{i j}^{A} \quad \text { in } A, \quad \text { and } \quad[b]_{i j}^{B}:=b \otimes e_{i j}^{B} \quad \text { in } B,
$$

and, in what follows, will freely use the identification:

$$
e_{i j}^{A}=1 \otimes e_{i j}^{A}=\left[1_{A_{0}}\right]_{i j}^{A}, \quad e_{i j}^{B}=1 \otimes e_{i j}^{B}=\left[1_{B_{0}}\right]_{i j}^{B} .
$$

Lemma 7.1. We have

$$
\begin{gathered}
{\left[a_{1}\right]_{i j}^{A}\left[a_{2}\right]_{k \ell}^{A}=\delta_{j k}\left[a_{1} a_{2}\right]_{i \ell}^{A}, \quad\left[b_{1}\right]_{i j}^{B}\left[b_{2}\right]_{k \ell}^{B}=\delta_{j k}\left[b_{1} b_{2}\right]_{i \ell}^{B} ;} \\
e_{i j}^{A}[a]_{k \ell}^{A}=\delta_{j k}[a]_{i \ell}^{A}=[a]_{i j}^{A} e_{k \ell}^{A} \\
e_{i j}^{B}[b]_{k \ell}^{B}=\delta_{j k}[b]_{i \ell}^{B}=[b]_{i j}^{B} e_{k \ell}^{B} \\
{[a]_{i j}^{A *}=\left[a^{*}\right]_{j i}^{A}, \quad[b]_{i j}^{B *}=\left[b^{*}\right]_{j i}^{B} .}
\end{gathered}
$$

Lemma 7.2. The von Neumann algebra $B$ is generated by $D_{0} \otimes \mathbf{C} 1$ and partial isometries $\left[u^{n}\right]_{i 1}^{B}, n \in \mathbb{Z}, i=2,3 \ldots$

Set $u(n, i):=e_{1 i}^{A} \cdot\left[u^{n}\right]_{i 1}^{B}, n \in \mathbb{Z}, i=2,3, \ldots$, a unitary in $p M p$.

Lemma 7.3. We have, for each $n \in \mathbb{Z}, i=2,3, \ldots$,

$$
E_{D}^{M}\left(u(n, i)^{k}\right)=0
$$

whenever $k \neq 0(\in \mathbb{Z})$.

Proof. One may and do assume $k>0$ since $E_{D}^{M}\left(u(n, i)^{-k}\right)=E_{D}^{M}\left(u(n, i)^{k}\right)^{*}$. Notice that

$$
\begin{cases}E_{D}^{M}\left(e_{1 i}^{A}\right)=E_{D}^{A}\left(e_{1 i}^{A}\right)=0 & \text { as long as } i \neq 1 \\ E_{D}^{M}\left(\left[u^{n}\right]_{i 1}^{B}\right)=E_{D}^{B}\left(u^{n} \otimes e_{i 1}^{B}\right)=\delta_{i 1} \cdot E_{D_{0}}^{B_{0}}\left(u^{n}\right)=0 & \text { as long as } i \neq 1\end{cases}
$$

Therefore, by the freeness, we have, for $n \in \mathbb{Z}, i \neq 1$,

$$
E_{D}^{M}\left(u(n, i)^{k}\right)=E_{D}^{M}\left(e_{1 i}^{A} \cdot\left[u^{n}\right]_{i 1}^{B} \cdots e_{1 i}^{A} \cdot\left[u^{n}\right]_{i 1}^{B}\right)=0 .
$$

Hence we are done.

We define the faithful normal conditional expectation

$$
\left(E_{D}^{M}\right)_{p}:=\left.E_{D}^{M}\right|_{p M p}: p M p \rightarrow D p=D_{0} \otimes \mathbf{C} p
$$

(which is well-defined since $p$ is in the smaller algebra $D$ ).

Lemma 7.4. The family

$$
\left\{A_{0} \otimes \mathbf{C} p\right\} \cup\{u(n, i): n \in \mathbb{Z}, i=2,3, \ldots\}
$$

is free with amalgamation over $D_{0} \otimes \mathbf{C} p$ with respect to $\left(E_{D}^{M}\right)_{p}$. 
Proof. Since all the $u(n, i)$ 's normalize the subalgebra $D_{0} \otimes \mathbf{C} p$ and since $\left(D_{0} \otimes\right.$ $\mathbf{C} p)\left(A_{0}^{\circ} \otimes \mathbf{C} p\right)$ and $\left(A_{0}^{\circ} \otimes \mathbf{C} 1\right)\left(D_{0} \otimes \mathbf{C} 1\right)$ are contained in $\left(A_{0}^{\circ} \otimes \mathbf{C} p\right)$, it suffices to show that

$$
\begin{aligned}
E_{D}^{M}\left(\left[a_{1}\right]_{11}^{A} u\left(n_{1}, i_{1}\right)^{k_{1}}\left[a_{2}\right]_{11}^{A} u\left(n_{2}, i_{2}\right)^{k_{2}}\right. & \\
& \left.\cdots\left[a_{m}\right]_{11}^{A} u\left(n_{m}, i_{m}\right)^{k_{m}}\left[a_{m+1}\right]_{11}^{A}\right)=0
\end{aligned}
$$

whenever all $k_{j}$ 's are not equal to 0 , the beginning and the ending letters $a_{1}, a_{m+1}$ are the identity 1 or in $A_{0}^{\circ}:=\operatorname{Ker} E_{D_{0}}^{A_{0}}$, and the other $a_{j}$ 's are

$$
a_{j} \text { is } \begin{cases}\text { in } A^{\circ} \text { or the identity } 1 & \text { if }\left(n_{j-1}, i_{j-1}\right) \neq\left(n_{j}, i_{j}\right), \\ \text { in } A_{0}^{\circ} & \text { if }\left(n_{j-1}, i_{j-1}\right)=\left(n_{j}, i_{j}\right) .\end{cases}
$$

We have, for $a \in A$,

$$
\begin{aligned}
& u\left(n_{1}, i_{1}\right)^{-k_{1}} \cdot[a]_{11}^{A} u\left(n_{2}, i_{2}\right)^{k_{2}} \\
& \quad=\left[u^{-n_{1}}\right]_{1 i_{1}}^{B} e_{i_{1} 1}^{A} \cdots\left[u^{-n_{1}}\right]_{i_{1}}^{B}[a]_{i_{1} i_{2}}^{A}\left[u^{n_{2}}\right]_{i_{2} 1}^{B} \cdots e_{1 i_{2}}^{A} \cdot\left[u^{n_{2}}\right]_{i_{2} 1}^{B}, \\
& u\left(n_{1}, i_{1}\right)^{k_{1}}[a]_{11} u\left(n_{2}, i_{2}\right)^{-k_{2}} \\
& \quad=e_{1 i_{1}}^{A}\left[u^{n_{1}}\right]_{i_{1} 1}^{B} \cdots e_{1 i_{1}}^{A}\left[u^{n_{1}}\right]_{i_{1} 1}^{B}[a]_{11}^{A}\left[u^{-n_{2}}\right]_{{ }_{1 i_{2}}}^{B} e_{i_{2} 1}^{A} \cdots\left[u^{-n_{2}}\right]_{1 i_{2}}^{B} e_{i_{2} 1}^{A},
\end{aligned}
$$

Thus, if $k_{1}, k_{2} \neq 0$ and if $a$ is such as in (7.5), we see that

$$
\begin{aligned}
u\left(n_{1}, i_{1}\right)^{-k_{1}}[a]_{11}^{A} u\left(n_{2}, i_{2}\right)^{k_{2}} & \in\left[u^{-n_{1}}\right]_{1 i_{1}}^{B} \underbrace{A^{\circ} \cdots A^{\circ}}_{\text {alternating }}\left[u^{n_{2}}\right]_{i_{2} 1}^{B} \\
& \subseteq B^{\circ} A^{\circ} \cdots A^{\circ} B^{\circ}, \\
u\left(n_{1}, i_{1}\right)^{k_{1}}[a]_{11} u\left(n_{2}, i_{2}\right)^{-k_{2}} & \in u\left(n_{1}, i_{1}\right) \underbrace{A^{\circ} \cdots A^{\circ}}_{\text {alternating }} u\left(n_{2}, i_{2}\right)^{*} \\
& \subseteq A^{\circ} \cdot B^{\circ} \cdot A^{\circ} \cdots A^{\circ} \cdot B^{\circ} \cdot A^{\circ} .
\end{aligned}
$$

Notice that

$$
\begin{gathered}
{\left[u^{n_{1}}\right]_{1 i_{1}}^{B}\left[u^{-n_{2}}\right]_{1 i_{2}}^{B}=\left[u^{n_{1}-n_{2}}\right]_{i_{1} i_{2}}^{B} \in B^{\circ},} \\
u\left(n_{1}, i_{1}\right)^{*} u\left(n_{2}, i_{2}\right)= \begin{cases}{\left[u^{-n_{1}}\right]_{1 i_{1}}^{B} e_{i_{1} i_{2}}^{A}\left[u^{n_{2}}\right]_{i_{2} 1}^{B} \in B^{\circ} A^{\circ} B^{\circ}} & \left(i_{1} \neq i_{2}\right), \\
{\left[u^{n_{2}-n_{1}}\right]_{11}^{B} \in B^{\circ}} & \left(i_{1}=i_{2}\right)\end{cases}
\end{gathered}
$$

as long as $\left(n_{1}, i_{1}\right) \neq\left(n_{2}, i_{2}\right)$, and one can easily check the desired equality (7.4) based on the above facts.

Thanks to Lemma 7.2 together with [V1, 3.1.Lemma], we see that the reduced von Neumann algebra $p M p$ is generated by

$$
\begin{aligned}
e_{1 i}^{A} \cdot(a \otimes 1) \cdot e_{j 1}^{A} & =\delta_{i j} \cdot(a \otimes p), \quad a \in A ; \\
e_{1 i}^{A} \cdot e_{k \ell}^{A} \cdot e_{j 1}^{A} & =\delta_{i k} \cdot \delta_{\ell j} \cdot p ; \\
e_{1 i}^{A} \cdot\left[u^{n}\right]_{k 1}^{B} \cdot e_{j 1}^{A} & =\delta_{i k} \cdot \delta_{j 1} \cdot u(n, i), \quad n \in \mathbb{Z}, i=2,3, \ldots
\end{aligned}
$$


We set

$$
N(n, i):=\left\{D_{0} \otimes \mathbf{C} p, u(n, i)\right\}^{\prime \prime} \cong D_{0} \rtimes_{\alpha^{n}} \mathbb{Z} \quad \text { (thanks to Lemma 7.3) }
$$

with the conditional expectation

$$
E_{(n, i)}=\left.E_{D}^{M}\right|_{N(n, i)}: N(n, i) \rightarrow D_{0} \otimes \mathbf{C} p \cong D_{0},
$$

which coincides with the canonical one from $D_{0} \rtimes_{\alpha^{n}} \mathbb{Z}$ onto $D_{0}$.

Summing up the discussions above, we conclude

Theorem 7.5. We have

$$
\left(p M p,\left(E_{D}^{M}\right)_{p}\right) \cong\left(A_{0}, E_{D_{0}}^{A_{0}}\right) *_{D_{0}}\left(\underset{\substack{n \in \mathbb{Z} \\ i=2,3, \ldots}}{*}\left(N(n, i), E_{(n, i)}\right)\right)
$$

Here, the amalgamated free product

$$
\underset{\substack{n \in \mathbb{Z} \\ i=2,3, \ldots}}{*}\left(N(n, i), E_{(n, i)}\right)
$$

is noting less than the crossed product of $D_{0}$ by the free group $\mathbb{F}_{\infty}$ with countably many generators, whose action is defined as follows:

$$
(\mathrm{Id})^{* \mathbb{N}} *(\alpha)^{* \mathbb{N}} *\left(\alpha^{2}\right)^{* \mathbb{N}} * \cdots *\left(\alpha^{n}\right)^{* \mathbb{N}} * \cdots .
$$

Here, $(\beta)^{* \mathbb{N}}$ means the free product of countably infinite copies of an automorphism $\beta$.

We further suppose that $A=B$, that is, $A_{0}=B_{0}=D_{0} \rtimes_{\alpha} \mathbb{Z}$. Theorem 7.5 says that the reduced von Neumann algebra $p M p$ is isomorphic to the crossed product of $D_{0}$ by the free group $\mathbb{F}_{\infty}$ whose action is

$$
\begin{aligned}
& \alpha *\left((\mathrm{Id})^{* \mathbb{N}} *(\alpha)^{* \mathbb{N}} *\left(\alpha^{2}\right)^{* \mathbb{N}} * \cdots *\left(\alpha^{n}\right)^{* \mathbb{N}} * \cdots\right) \\
& =(\mathrm{Id})^{* \mathbb{N}} *(\alpha)^{* \mathbb{N}} *\left(\alpha^{2}\right)^{* \mathbb{N}} * \cdots *\left(\alpha^{n}\right)^{* \mathbb{N}} * \cdots .
\end{aligned}
$$

Here, this equality follows from the simple fact: $\alpha *(\alpha)^{* \mathbb{N}}=(\alpha)^{* \mathbb{N}}$.

Remark 7.6. The result obtained in this section is thought of as a negative evidence towards generalizing the work $[\mathrm{G}]$ on the invariant "cost" of D. Gaboriau to general non-singular discrete measured groupoids. Roughly speaking, the "cost" counts the number of free components in a given finite-measure preserving countable equivalence relation, and recently D. Shlyakhtenko [S2] generalized further to finite-measure preserving discrete groupoids from the free entropic viewpoint. Our result here says that the number of free components cannot be determined in the general non-singular case. Indeed, we suppose that our $A=B$ is a factor of type III (or of type $\mathrm{II}_{\infty}$ ) and that $D$ is a Cartan subalgebra as before. Then 
the amalgamated free product $M$ is also a factor of type III and captured as a groupoid von Neumann algebra (see $[\mathrm{Ks}]$ ). We can then choose an isometry $v \in A$ in such a way that $v D v^{*}=D p$ with $v v^{*}=p \in D$. The $\mathrm{Ad} v$ gives rise to an isomorphism between $M \supseteq D$ and $p M p \supseteq D p$. Moreover, we can show

$$
\left(E_{D}^{M}\right)_{p} \circ \operatorname{Ad} v=\operatorname{Ad} v \circ E_{D}^{M}
$$

and hence $\left(M \supseteq D, E_{D}^{M}\right)$ can be identified with $\left(p M p \supseteq D p,\left(E_{D}^{M}\right)_{p}\right)$, and the former has two free components, but the latter has infinite ones.

The discussions here (with trivial changes) also implies

Corollary 7.7. Let $N$ be an infinite injective factor of non-type I with a Cartan subalgebra $D$. Then we have

$$
N *_{D} N \cong N *_{D} N *_{D} N \cong \cdots \cong N *_{D} N *_{D} N *_{D} \cdots
$$

Remark 7.8. One may replace $B\left(\ell^{2}(\mathbb{N})\right)$ by $k \times k$ matrix algebra $M_{k}(\mathbf{C})$ in the setting, and the discussion here still works without any essential change and the assertion (7.6) should be changed to

$$
\left(p M p,\left(E_{D}^{M}\right)_{p}\right) \cong\left(A_{0}, E_{D_{0}}^{A_{0}}\right) *_{D_{0}}\left(\underset{\substack{n \in \mathbb{Z} \\ i=2,3, \ldots, k}}{*}\left(N(n, i), E_{(n, i)}\right)\right)
$$

so that if $A_{0}=B_{0}$ then $p M p$ is the crossed-product of $D_{0}$ by the free group $\mathbb{F}_{\infty}$ whose action is:

$$
(\mathrm{Id})^{*(k-1)} *(\alpha)^{* k} *\left(\alpha^{2}\right)^{*(k-1)} * \cdots *\left(\alpha^{n}\right)^{*(k-1)} * \cdots .
$$

This is in particular thought of as a reduction formula of the amalgamated free product $R *_{D} R$ of two copies of the injective $\mathrm{II}_{1}$ factor $R$ over a common Cartan subalgebra $D$ thanks to $[\mathrm{CFW}$. The result says that, if the amalgamated free product $R *_{D} R$ had the whole fundamental group (defined as in [P1]) $\mathcal{F}\left(R *_{D} R \supseteq\right.$ $D)=\mathbf{R}_{+}^{\times}$, then the number of its free components would not be able to be determined uniquely. This is completely analogous to the situation of free group factors $L\left(\mathbb{F}_{n}\right)$ with finite $n$ (see [V2, 6.13 Remark]). This analogy is very natural in a certain sense, because the amalgamated free product $R *_{D} R$ can be regarded as one candidate of the true generalizations of the free group factor $L\left(\mathbb{F}_{2}\right)$ from the view-point of the idea generalizing the group von Neumann algebra construction to the group-measure space construction. (D. Shlyakhtenko [S1] provided another candidate "A-valued semicircular systems" from the view-point of Voiculecu's free Gaussian functor (see $[\mathrm{VDN}])$.) 


\section{REFERENCES}

[A] S. Adams, Boundary amenability for word hyperbolic groups and an application to smooth dynamics of simple groups. Topology 33 (1994), no. 4, 765-783.

[BD] E.F. Blanchard \& K.J. Dykema, Embeddings of reduced free products of operator algebras. preprint (1999).

[C1] A. Connes, Une classification des facteurs de type III. Ann. Scient. Èc. Norm. Sup. 8 (1973), 133-252.

[C2] A. Connes, Classification of injective factors. Cases $\mathrm{II}_{1}, \mathrm{II}_{\infty}, \mathrm{III}_{\lambda}, \lambda \neq 1$. Ann. of Math. 104 (1976), 73-115.

[CFW] A. Connes, J. Feldman \& B. Weiss, An amenable equivalence relation is generated by a single transformation. Ergod. Th. \& Dynam. Sys. 1 No. 4 (1982), 431-450.

[CT] A. Connes \& M. Takesaki, The flow of weights on factor of type III. Tôhoku Math. Journ., 29 (1977), 473-575.

[EFW] M. Enomoto, M. Fujii \& Y. Watatani, KMS states for gauge action on $\mathcal{O}_{A}$. Math. Japon. 29 (1984), no. 4, 607-619.

[FM] J. Feldman \& C.C. Moore, Ergodic equivalence relations, cohomology, and von Neumann algebras. I, II. Trans. Amer. Math. Soc., 234 (1977), 289-324, $325-358$.

[G] D. Gaboriau, Coût des relations d'équivalence et des groupes. Invent. Math. 139 (2000), no. 1, 41-98.

[HOO] T. Hamashi, Y. Oka \& M. Osikawa, Flows associated with ergodic nonsingular transformation groups. Publ. RIMS, Kyoto Univ. 11 (1975), 31-50.

[Kr] W. Krieger, On ergodic flows and the isomorphism of factors. Math. Ann. 223 (1976), 19-70.

[Ks] H. Kosaki, Free products of measured equivalence relations, Preprint. (2001)

[KS] G. Kuhn \& T. Steger, More irreducible boundary representations of free groups. Duke Math. J. 82 (1996), no. 2, 381-436.

[P1] S. Popa, Some rigidity results in type $\mathrm{II}_{1}$ factors. C. R. Acad. Sci. Paris Sér. I, Math. 311 (1990), no. 9, 535-538.

[P2] S. Popa, Markov traces on universal Jones algebras and subfactors of finite index. Invent. Math. 111 (1993), 375-405. 
[PS] C. Pensavalle \& T. Steger, Tensor products with anisotropic principal series representations of free groups. Pacific J. Math. 173 (1996), no. 1, 181-202.

[RR] J. Ramagge \& G. Robertson, Factors from trees. Proc. Amer. Math. Soc. 125 (1997), no. 7, 2051-2055.

[S1] D. Shlyakhtenko, $A$-valued semicercular systems. J. Funct. Annal. 166 (1999), 1-47.

[S2] D. Shlyakhtenko, Microstates free entropy and cost of equivalence relations. preprint (1999).

[Sp] J. Spielberg, Free-product groups, Cuntz-Krieger algebras, and covariant maps. Intern. J. Math. 2 (1991), 457-476.

[T1] M. Takesaki, Conditional expectations in von Neumann algebras. J. Funct. Annal. 9 (1972), 306-321.

[T2], M. Takesaki, Duality for crossed product and the structure of von Nenmann algebras of type III. Acta Math. 131 (1973), 249-310.

[U1] Y. Ueda, Amalgamated free product over Cartan subalgebra. Pacific J. Math. 191, No.2 (1999), 359-392.

[U2] Y. Ueda, Fullness, Connes' $\chi$-groups, and ultra-products of amalgamated free products over Cartan subalgebras. Trans. Amer. Math. Soc. 355 (2003), 349-371 .

[Ver] A. M. Vershik, Trajectory Theory, chap. 5 in Dynamical Systems II, Ya. G. Sinai, ed. (Trandlated from Russian) (ENS) Encyclopaedia of Mathematical Sciences (Springer-Verlag, Berlin) 2, 77-92 (1989)

[V1] D. Voiculescu, Circular and semicircular systems and free product factors. Operator algebras, unitary representations, enveloping algebras, and invariant theory (Paris, 1989), 45-60, Progr. Math. 92, Birkhäuser Boston, Boston, MA, 1990.

[V2] D. Voiculescu, The analogues of entropy and Fisher's information measure in free probability theory, III: The absence of Cartan subalgebras. Geometric and Functional Analysis 6 (1996), 172-199.

[VDN] D.-V. Voiculescu, K.-J. Dykema \& A. Nica, Free Random Variables. CRM Monograph Series I, Amer. Math. Soc. Providence, RI, 1992. 
[Z] R.J. Zimmer, Ergodic theory and semisimple groups. Monographs in Mathematics, 81. Birkhäuser Verlag, Basel-Boston, Mass., 1984.

Department of Mathematics, Graduate School of Science, Hiroshima UniverSity, HigASHI-HiRoshima, 739-8526, JAPAN

Current Address: Faculty of Mathematics, Kyushu University, Fukuoka, 8108560, JAPAN

E-mail address: e-mail: ueda@math.kyushu-u.ac.jp 